\title{
Fonctionnement Hydrique du Baobab (Adansonia digitata L.) en Moyenne et Haute Casamance (Sénégal)
}

\author{
Ababacar Ndiaye, \\ Laboratoire d'Agroforesterie et d'Ecologie/URF Sciences et Technologies \\ (Université Assane Seck de Ziguinchor/Sénégal) \\ Centre National de Recherches Forestières \\ (Institut Sénégalais de Recherches Agricoles/Sénégal) \\ Tamsir Mbaye, \\ (Chargé de Recherches, $\mathrm{PhD}$, Phytogéographie) \\ Institut Sénégalais de Recherches Agricoles/Sénégal \\ Daouda Ngom, \\ (Professeur Assimilé, PhD, Ecologie) \\ Université Cheikh Anta Diop/Sénégal \\ Mamadou Ousseyni Ly, \\ (Chargé de recherches, $\mathrm{PhD}$, Ecophysiologie) \\ Institut Sénégalais de Recherches Agricoles/Sénégal \\ Diegane Diouf, \\ (Professeur Titulaire, $\mathrm{PhD}$, Physiologie) \\ Université Cheikh Anta Diop/Sénégal
}

Doi: 10.19044/esj.2019.v15n3p292～URL:http://dx.doi.org/10.19044/esj.2019.v15n3p292

\section{Résumé}

Cette étude aborde le fonctionnement hydrique du baobab afin de mieux appréhender ses relations en eau avec ses phases phénologiques et l'effet induit par les saisons climatiques et de celui de la toposéquence. Pour cela, l'humidité du sol, la teneur relative en eau (TRE) et le potentiel hydrique foliaire $(\Psi)$ ont été étudiés. Les données ont été collectées sur deux sites, en plateau et dans le bas-fond, en saison sèche chaude (SSC), en pleine saison des pluies (PSP) et en saison sèche fraiche (SSF). La SSC correspond à l'apparition des premières feuilles. La PSP correspond à la pleine feuillaison et la fin de la floraison et au début de la fructification. La SSF correspond à la maturation avancée des fruits et à la pleine chute des feuilles. Le logiciel $\mathrm{R}$ version 3.4.2 a été utilisé pour traiter les données. Il a permis d'effectuer des tests pour l'analyse des données. Les résultats montrent une variation saisonnière de l'humidité du sol autour de la zone racinaire du baobab. En 
effet, en SSC, l'humidité du sol est à son niveau le plus faible contrairement en MSP et en SSF. Cependant, la faible disponibilité en eau du sol en SSC n'empêche pas l'état hydrique interne du baobab d'être élevé. En effet, espèce caduque à tronc succulent, les $\Psi$ et les TRE trouvés du baobab sont plus élevés en saison sèche qu'en saison pluvieuse. Egalement, en fonction des saisons climatiques, les différences obtenues sur les $\Psi$ d'une part, et sur les TRE d'autre part, sont statistiquement significatives $(\mathrm{P}<0,05)$ sur le plateau contrairement au bas-fond. Ce dernier bénéficie des conditions hydriques du sol plus favorables pendant la saison sèche, ce qui a probablement minimisé ces différences, d'où le rôle important de la toposéquence sur la recharge de l'eau du sol. Ces résultats sont importants dans le but de mieux encourager la propagation les plantations de baobab grâce à sa capacité d'adaptation aux saisons climatiques et au milieu, et de sa tolérence à la sècheresse saisonnière du sol, afin de palier la dégradation de la ressource et faire face aux effets du changement climatique.

Mots clés: Adansonia digitata L., fonctionnement hydrique, phases phénologiques, toposéquence et saisons climatiques, Moyenne et Haute Casamance 


\title{
Water Functioning of Baobab (Adansonia digitata L.) in Middle and Upper Casamance (Senegal)
}

\author{
Ababacar Ndiaye, \\ Laboratoire d'Agroforesterie et d'Ecologie/URF Sciences et Technologies \\ (Université Assane Seck de Ziguinchor/Sénégal) \\ Centre National de Recherches Forestières \\ (Institut Sénégalais de Recherches Agricoles/Sénégal) \\ Tamsir Mbaye, \\ (Chargé de Recherches, $\mathrm{PhD}$, Phytogéographie) \\ Institut Sénégalais de Recherches Agricoles/Sénégal \\ Daouda Ngom, \\ (Professeur Assimilé, PhD, Ecologie) \\ Université Cheikh Anta Diop/Sénégal \\ Mamadou Ousseyni Ly, \\ (Chargé de recherches, $\mathrm{PhD}$, Ecophysiologie) \\ Institut Sénégalais de Recherches Agricoles/Sénégal \\ Diegane Diouf, \\ (Professeur Titulaire, $\mathrm{PhD}$, Physiologie) \\ Université Cheikh Anta Diop/Sénégal
}

\begin{abstract}
This study deals with the baobab's water functioning in order to better understand its water relations with its phenological phases and the effect induced by the climatic seasons and that of the toposequence. For this, the soil moisture, the relative water content (TRE) and the leaf water potential ( $\Psi$ ) were studied. Data were collected at two sites, in the upland and in the lowland, in the hot dry season (SSC), in the middle of the rainy season (PSP) and in the cool dry season (SSF). SSC corresponds to the appearance of the first leaves. The PSP corresponds to full leafing and the end of flowering and early fruiting. SSF is the advanced ripening of fruits and the full fall of leaves. The $\mathrm{R}$ version 3.4.2 software was used to process the data. It allowed to test the data analysis. The results show a seasonal variation of soil moisture around the baobab root zone. Indeed, in SSC, soil moisture is at its weakest level, unlike in MSP and SSF. However, the low water availability of soil in SSC does not prevent the internal water condition of baobab from being high. Indeed, succulent trunk deciduous species, $\Psi$ and TRE found baobab are higher in the dry season than in the rainy season. Also, according to the
\end{abstract}


climatic seasons, the differences obtained on $\Psi$ on the one hand, and on the TREs on the other hand, are statistically significant $(\mathrm{P}<0.05)$ on the upland contrary to the lowland. The latter benefits from more favorable soil moisture conditions during the dry season, which probably minimized these differences, hence the important role of toposequence on groundwater recharge. These results are important in order to better encourage the spread of baobab plantations through its ability to adapt to the climatic seasons and the environment, and its tolerance of seasonal soil drought, in order to mitigate the degradation of the resource and cope with the effects of climate change.

Keywords: Adansonia digitata L., water regulation, phenological phases, toposequence and climatic seasons, Middle and Upper Casamance

\section{Introduction}

L'irrégularité de la pluviométrie due aux changements climatiques et la forte pression exercée sur les espèces forestières d'importance économique ont comme conséquence une régression des peuplements naturels. Au Sénégal, cette régression est notée sur des espèces fruitières forestières (Diagne, 2000) et plus particulièrement sur les populations de baobab (Collière, 2002 ; Ndiaye, 2013). Cette espèce joue un rôle important dans l'alimentation et le revenu des ménages en milieu rural notamment les jeunes et les femmes qui sont plus vulnérables aux effets du changement climatique. Ainsi, dans le souci de renforcer la sécurité alimentaire et le bien être des ménages ruraux à travers des actions d'amélioration des plantes et de maintien de la biodiversité végétale, des tentatives de réhabilitation et de rajeunissement des parcs à baobabs et de domestication ont été entreprises dans certains pays d'Afrique de l'Ouest. Au Sénégal, des tests de greffage horticole et d'inoculation mycorhizienne du baobab ont été effectués pour améliorer sa croissance (Samba et al., 2003). Au Burkina Faso, il y a eu des tests d'introduction de jeunes baobabs en station et en milieu rural dans le Plateau Central (Bationo et al., 2009) afin de rajeunir et de reconstituer les parcs. En ce qui concerne la domestication, des études ont été faites au Mali et au Sénégal sur l'adoption des cultures maraichères de feuilles de baobab (Savard, 2003, Cissé 2015) pour réduire la pression sur les grands baobabs des parcs.

Seulement, quelle que soit l'action menée, il n'en demeure pas moins que la réussite d'un tel défi impliquerait nécessairement une maitrise du fonctionnement hydrique de l'espèce. Ainsi, des études récentes sur la réponse des baobabs face à la sècheresse hydrique du sol ont été entreprises sur de jeunes sujets (Randriamanana et al., 2012; Van den Bilcke et al., 2013; Bouda et al., 2015; Mukhtar, 2016 etc.). Il s'agissait d'évaluer l'effet de la sécheresse sur les paramètres de croissance (Bouda et al., 2015 ; Mukhtar, 2016), sur le flux de sève et entre autres sur la stratégie d'utilisation de l'eau (Van den 
Bilcke et al., 2013; Randriamanana et al., 2012). Parmi les résultats, certains auteurs ont montré que le baobab réagit différemment par rapport aux dégrés de stress hydrique au sol. Ceci suggère que l'espèce développe des stratégies par rapport à la disponibilité hydrique du sol. Par ailleur Korbo et al. (2013), en étudiant la production foliaire de jeunes plantes de baobab ont trouvé une faible production de feuilles en saison sèche (la période de défeuillaison des baobabs adultes) malgré l'irrigation. Cela laisse à supposer que les mécanismes de croissance ne dépendent pas seulement de la disponibilité de l'eau, mais, aussi, d'autres paramétres climatiques. De plus, il est à noter que l'environnement physique détermine les contraines que subit un arbre. Ainsi, la topographie est un élément structurant important. Elle peut influencer sur la disponibilité en eau pendant les périodes sèche (Jabot, 2009). D'ou l'hypothèse selon laquelle les diffénts niveaux topographiques (plateau, basfond) induisent une différence sur l'état hydrique interne du baobab.

Cependant, il a été noté de rares études sur les relations hydrides des espèces de baobabs adultes, dans leur milieu naturel, dont Adansonia digitata L. n'y figure pas (Chapotin et al., 2006a,b,c) à l'exception des travaux de Fenner (1980). Ce dernier a étudié le potentiel hydrique de Adansonia digitata L. et ses pertes en eau par abscission. Par contre l'effet des saisons climatiques et la toposéquence sur la variation des paramètres hydriques n'ont pas été prise en compte.

C'est dans ce contexte que cette étude a été entreprise sur Adansonia digitata L. dans les parcs naturels de la Moyenne et Haute Casamance du Sénégal afin d'évaluer la variation de l'état hydrique interne du baobab adulte et l'effet induit par les saisons climatiques et la toposéquence.

\section{Matériel et méthodes \\ Zone d'études}

L'étude a été menée dans les parcs à baobabs des villages de Dianabo et Bougnadou situés respectivement dans les régions de Kolda (commune de Saré Bidji) et de Sédhiou (commune de Sakar) (figure 1). Ces deux régions administratives couvrent respectivement les parties Haute et Moyenne Casamance, une zone agro-écologique du Sénégal. 


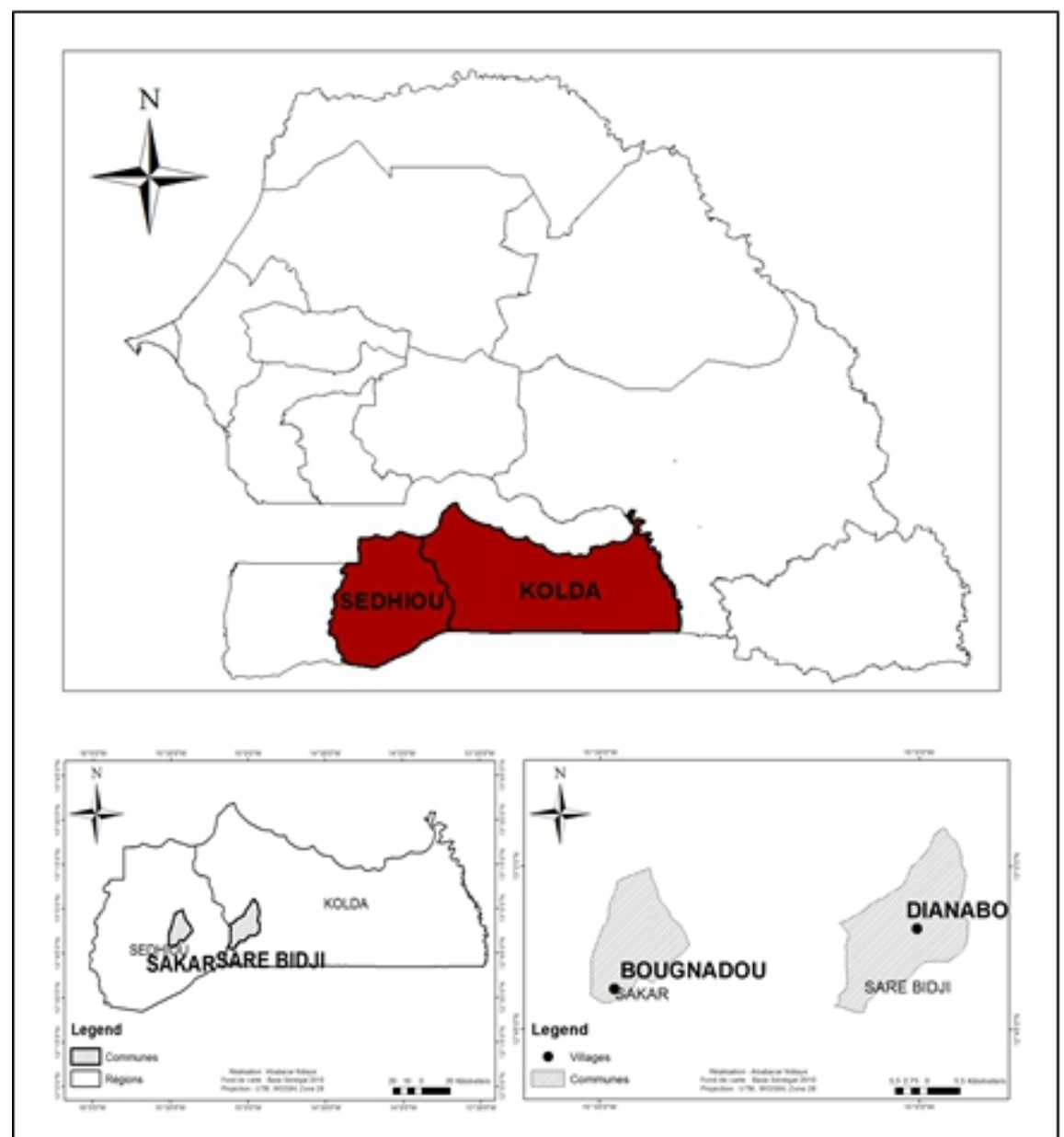

Figure 1 : carte de localisation des sites d'étude Bougnadou et Dianabo en Moyenne (Sédhiou) et Haute Casamance (Kolda)

Cette zone est caractérisée par un climat du domaine sud-soudanien continental (Sagna, 2005) avec une pluviométrie moyenne annuelle de $1054 \pm 206 \mathrm{~mm}$ entre 1985 et 2016 indiquant une forte variabilité (figure 2). 


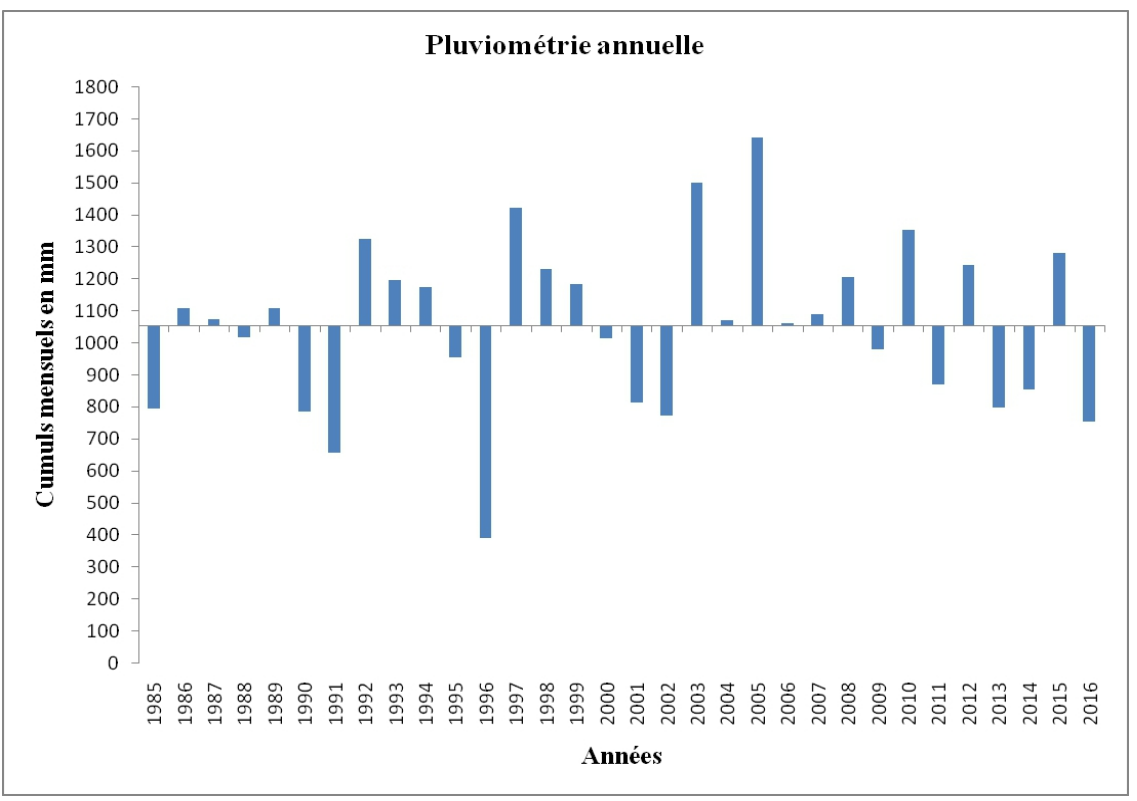

Figure 2: variation de la pluviométrie annuelle de la Moyenne et Haute Casamance de 1985 à 2016.

La Casamance possède une saison des pluies relativement courte (5 mois) et une longue saison sèche (7 mois) subdivisée en saison sèche fraiche (SSF) et en saison sèche chaude (SSC) (figure 3). Les températures sont en baisse entre le mois de novembre et février, correspondant à la SSF. Cependant, elles sont élevées durant la période allant de mars à mai, correspondant à la SSC, période au cours de laquelle, l'harmattan, vent chaud et sec, domine dans la zone. Cependant, du mois de juin au mois d'octobre, correspondant à la saison des pluies (SP), les températures diminuent sous l'influence des pluies.

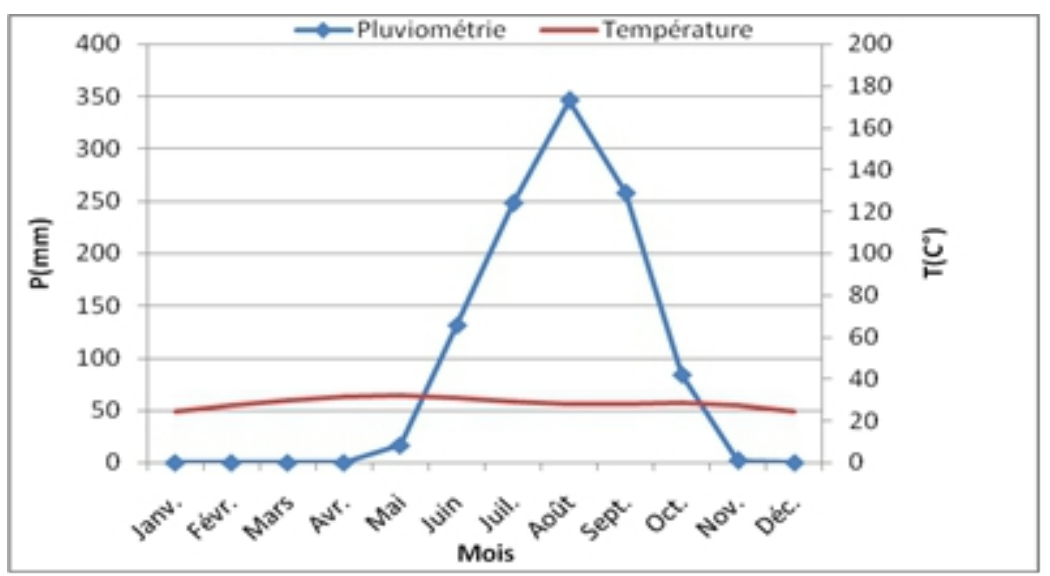

Figure 3: diagramme ombrothermique de la Moyenne et Haute Casamance de 1985 à 2015. 
La portion de végétation est du type savane herbeuse comportant une strate arborée dominée par de grands arbres caractéristiques du secteur soudano-guinéen. Il s’agit de Adansonia digitata L. (baobab), Daniellia oliveri (Rolfe) Huch. \& Dalz. (santan), Parkia biglobosa (Jacq.) R. Br. Ex G. Don (nété), Pterocarpus erinaceus Poir. (ven), Cordyla pinnata (Lepr. Ex A. Rich.) Milne-Readhead (dimb), Sterculia setigera Del. (mbep), Elaeis guineensis Jacq. (palmier à huile), Rhizophora racemosa G. Mey, Avicennia germinas L. etc. Ces espèces à usages multiples sont réparties en fonction de la toposéquence (Bas-fond, Plateau) et des systèmes d'utilisation des terres, formant ainsi par endroit des parcs tels que ceux à baobabs (Ndiaye, 2013).

\section{Méthodes d'études}

Le choix des sites a été influencé par la répartion des parcs à baobab en fonction de la toposéquence dans le paysage naturel de la Casamance (Ndiaye, 2013). Ainsi, les parcs de Dianabo (plateau) et de Bougnadou (basfond) ont constitué le site d'étude.

Dans chaque parc, trois baobabs adultes ont été choisis en tenant compte des travaux d'Achetar et al. (1991) et Bourou (2012) pour les observations et mesures au sein d'une population d'espèce végétale. Ces individus d'apparence morphologiquement semblable appartiennent à la même classe modale. Il s'agit de la classe de circonférence de $15-7 \mathrm{~m}$ ] dans le bas-fond et sur le plateau. Ainsi, les observations et mesures ont été effectuées en fonction de la combinaison des saisons climatiques avec les principales phases phénologiques du baobab. Il s'agissait de la SSC, au mois de mai qui correspond à l'apparition des feuilles, la PSP, en septembre, correspondant à la pleine feuillaison, à la fin de la floraison et au début de la fructification et enfin la SSF, en décembre, avec la maturation avancée des fruits et à la pleine chute des feuilles. Les mesures avaient porté sur des variables d'estimation de l'humidité du sol et de la détermination de l'état hydrique interne de la plante. L'humidité du sol a été estimée à travers la teneur en eau du sol, alors que l'état hydrique interne a été déterminé par le potentiel hydrique foliaire $(\Psi)$ et la teneur relative en eau (TRE).

\section{La teneur en eau du sol dans la zone d'extraction racinaire}

En tenant compte de l'enracinement superficiel de A. digitata L. (Fenner, 1980), les variations saisonnières de l'humidité du sol autour de la zone racinaire ont été déterminées sous et hors houppier des trois individus de baobab choisis dans chaque site.

Les profils hydriques ont été déterminés par la méthode gravimétrique. Les échantillons de sol ont été prélevés avec une tarière autour de chaque baobab suivant les quatre points cardinaux à des distances égales à la moitié du rayon du houppier $(1 / 2 \mathrm{R})$, au rayon du houppier $(\mathrm{R})($ échantillonnage 
composite horizontal) correspond au sous houppier et au trois demi du rayon du houppier (3/2 R) en hors couvert de l'arbre. Les prélèvements ont été faits jusqu'à $1 \mathrm{~m}$ de profondeur à des intervalles de $10 \mathrm{~cm}$ (échantillonnage ponctuel vertical). Ces échantillons de sol ont été pesés au frais puis séchés à l'étuve à $105^{\circ} \mathrm{C}$ jusqu' à la masse constante.

L'humidité pondérale (Hp), définie comme étant la valeur massique de l'eau contenue dans un échantillon de sol, a été déterminée à partir des masses fraiches (Mf) et sèches (Ms) du sol à partir de la formule suivante: $\mathrm{Hp}(\%)=[(\mathrm{Mf}-\mathrm{Ms}) / \mathrm{Ms}] * 100$

\section{Potentiel hydrique foliaire $(\Psi)$}

Le potentiel hydrique foliaire a été mesuré par la chambre à pression de Scholander. Les échantillons de feuilles ont été choisis parmi celles situées à une hauteur de la couronne où elles sont plus exposées au soleil (Bationo et al., 2001). Ainsi, trois types de potentiels ont été mesurés.

Le potentiel hydrique foliaire de base $(\Psi \mathrm{b})$ a été mesuré avant le lever du soleil, période pendant laquelle la transpiration est presque nulle. Il intègre le potentiel hydrique du sol dans la zone active du système racinaire. Le potentiel hydrique foliaire minimum $(\Psi \mathrm{m})$, mesuré au milieu de la journée, représente la contrainte hydrique journalière résultant du bilan hydrique entre le taux de transpiration et d'absorption indiquant ainsi le déficit hydrique interne maximal de la plante. Le potentiel hydrique foliaire de récupération ( $\Psi$ r), obtenu après le coucher du soleil, caractérise la réhydratation de la plante suite à un quasi arrêt des pertes d'eau par évapotranspiration.

\section{Teneur relative en eau (TRE)}

Elle est déterminée sur un raméal par arbre pris sur la même période de la journée (19h-20h) pendant les trois saisons climatiques étudiées, avec la formule suivante (Weatherley P.E., 1950 cité par Fahmi et al., 2011):

$\mathrm{TRE}=[(\mathrm{MF}-\mathrm{MS}) /(\mathrm{MT}-\mathrm{MS})] \times 100$

$\mathrm{MF}$ : Masse de la matière fraiche.

MT : Masse à la turgescence, obtenue après 24 heures de trempage dans de l'eau distillée et à l'obscurité.

MS : Masse sèche, obtenue après séchage à l'étuve à $70^{\circ} \mathrm{C}$ pendant 48 heures.

\section{Analyse des données}

L’analyse des données a été réalisée par le logiciel $\mathrm{R}$ version 3.4.2. Les distributions ne suivent pas la loi normale d'après le test Shapiro-Wilk. Le test MANOVA (Analyse de variance multivariée) de Roy et le test ANOVA (Analyse de variance) non paramétrique de Kruskal-Wallis par rangs au seuil de $95 \%$ ont été utilisés. Le classement en groupes homogènes $(a, a b, b)$ est obtenu grâce au test de Student-Newman-Keuls (SNK). 


\section{Resultats}

Variation saisonnière de l'humidité en eau du sol autour de la zone racinaire

Sur le bas-fond comme sur le plateau, l'humidité du sol augmente en suivant la profondeur du sol et les saisons climatiques. Elle progresse des couches superficielles vers les plus profondes. Egalement, l'écart entre les courbes indique que le sol est plus humide en SSF et en PSP qu'en SSC où il est moins humide (Figure 4).

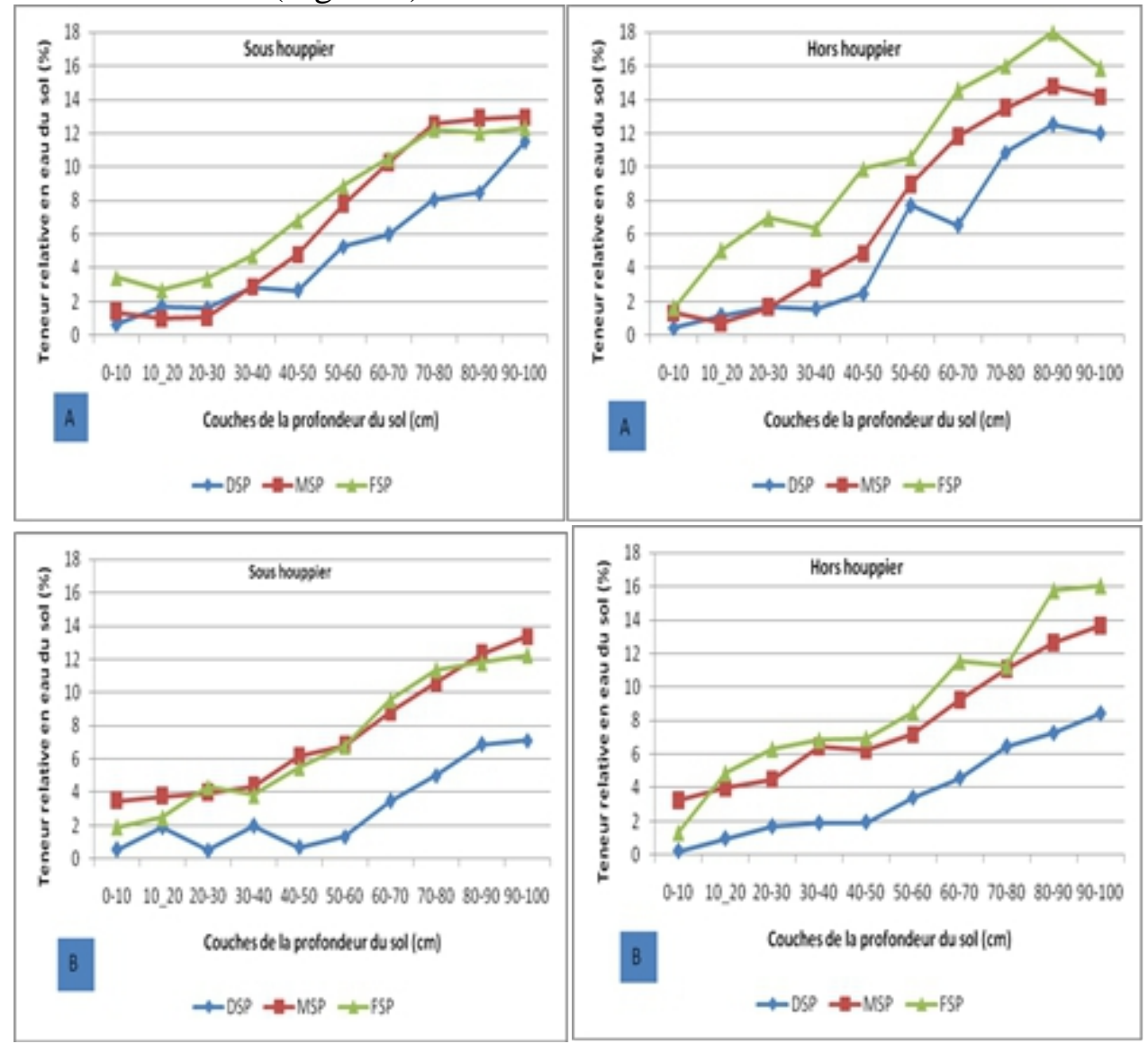

Figure 4: varaition saisonnière de l'humidité du sol sur le bas-fond (A) et sur le plateau (B).

PSP (pleine saison des pluies); SSF (saison sèche fraiche); SSC (saison sèche chaude). 


\section{Etat hydrique interne du baobab}

\section{Variation saisonnière de l'état hydrique interne}

Les $\Psi$ (surtout le $\Psi \mathrm{m}$ ) et la TRE ont des valeurs moyennes plus élevées en saison sèche comparée à la saison pluvieuse (figures 5 et 6).

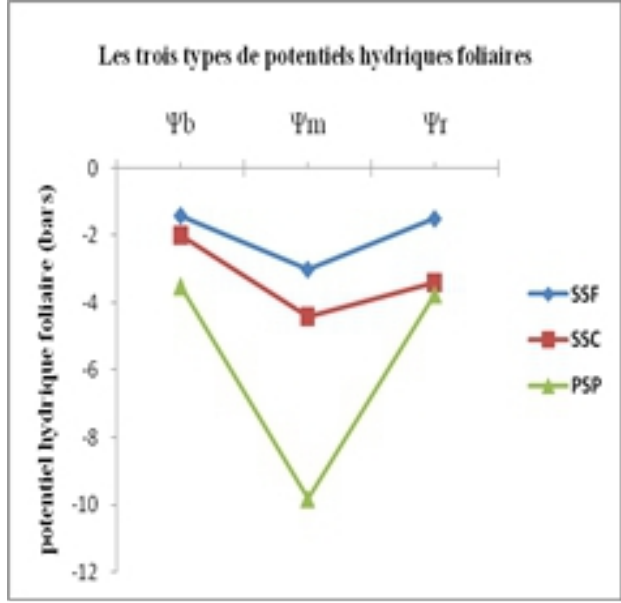

Figure 5 : variation saisonnière du $\Psi$

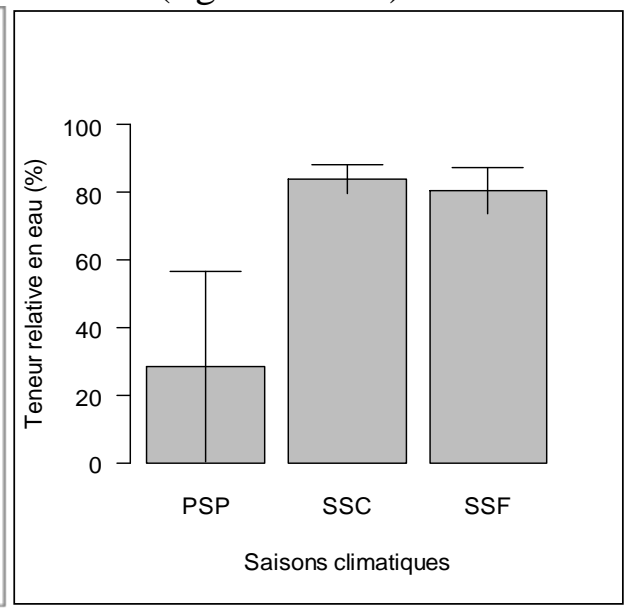

Figure 6: variation saisonnière de la TRE

\section{Effets des saisons climatiques sur la variation hydrique du baobab}

Il ressort des résultats du test MANOVA (Analyse de variance multivariée) que les saisons climatiques ont un effet significatif $(\mathrm{P}<0,05)$ sur l'ensemble des variables hydriques ( $\Psi$ et TRE). En revanche, tel n'est pas le cas pour la toposéquence et l'interaction entre toposéquence et les saisons climatiques où leurs effets ne sont pas significatifs (tableau 1).

Tableau 1: test MANOVA de l'effet des saisons climatiques et de la toposéquence ainsi que de leur interaction sur les variables hydriques.

\begin{tabular}{|c|c|c|c|c|c|c|}
\hline & Df & Roy & approx F & num Df & den Df & $\operatorname{Pr}(>$ F $)$ \\
\hline Toposéquence & 1 & 1.8822 & $\mathbf{0 . 8 3 6 5}$ & 9 & 4 & $\mathbf{0 . 6 2 3 5 5}$ \\
\hline Saisons & 2 & 9.3016 & 5.1675 & 9 & 5 & $0.04262 *$ \\
\hline Top. /Saisons & 2 & 5.2219 & 2.9011 & 9 & 5 & 0.12671 \\
\hline Residuals & 12 & & & & & \\
\hline
\end{tabular}

*: Différence significative ( $\mathrm{p}<0,05)$; Df: degré de liberté; Roy: valeur statistique du test de Roy; approx F: valeur statistique approximative F de la distribution F; num Df:degré de liberté du numérateur; den Df: degré de liberté du dénominateur; $\operatorname{Pr}(>\mathbf{F})=$ p-value (valeur de probabilité).

\section{Effets de la toposéquence sur la variation hydrique du baobab}

Malgré l'absence d'effet significatif de la toposéquence sur les variables hydriques (tableau 1), les résultats du tableau 2 montrent que l'effet du climat est significatif $(\mathrm{P}<0,05)$ uniquement sur les baobabs situés au niveau du plateau et non significative $(\mathrm{P}>0,05)$ sur le bas-fond. Pour les trois $\Psi$ 
mesurés ( $\Psi \mathrm{b}, \Psi \mathrm{m}$ et $\Psi \mathrm{r}$ ) et la TRE, les valeurs les plus élevées sont observées en saison sèche (SSC et SSF).

Tableau 2 : test ANOVA (Analyse de la variance) de Kruskal-Wallis des rangs de comparaison de chaque variable hydrique sur les saisons climatiques.

\begin{tabular}{|l|l|l|l|l|l|}
\hline Variables & Toposéquence/Saisons & SSC & PSP & SSF & Pr $(>$ F) \\
\hline \multirow{4}{*}{ TRE } & plateau & $6 \mathrm{a}$ & $2 \mathrm{~b}$ & $7 \mathrm{a}$ & $0.027 *$ \\
\cline { 2 - 7 } & bas-fond & 7.33 & 2.66 & 5.00 & 0.09454 \\
\hline \multirow{4}{*}{$\boldsymbol{\Psi m}$} & plateau & $7 \mathrm{a}$ & $2 \mathrm{~b}$ & $6 \mathrm{a}$ & $0.004096 * *$ \\
\cline { 2 - 7 } & bas-fond & 5 & 3 & 7 & 0.2088 \\
\hline \multirow{4}{*}{$\boldsymbol{\Psi r}$} & plateau & $6.33 \mathrm{a}$ & $2.00 \mathrm{~b}$ & $6.66 \mathrm{a}$ & $0.0177 *$ \\
\cline { 2 - 7 } & bas-fond & 5.16 & 2.83 & 7.00 & 0.1723 \\
\cline { 2 - 7 } & plateau & $5.00 \mathrm{ab}$ & $2.33 \mathrm{~b}$ & $7.66 \mathrm{a}$ & $0.0135 *$ \\
\hline
\end{tabular}

Les valeurs obsersées constituent les moyennes des rangs du test ANOVA de Kruskal-Wallis. $\mathbf{a}, \mathbf{a b}, \mathbf{b}$ et $\mathbf{c}$ : représentent des valeurs en groupes homogènes du test $\mathrm{SNK}$; * : Différence significative $(p<0,05)$; ** : Différence très significative $(p<0,01)$;

*** : Différence hautement significative $(\mathrm{p}<0,001) ; \operatorname{Pr}(>\mathbf{F})=\mathrm{p}$-value (valeur de probabilité); PSP (pleine saison des pluies); SSF (saison sèche fraiche); SSC (saison sèche chaude).

\section{Discussion}

\section{Variation saisonnière de la teneur en eau du sol dans la zone racinaire}

La connaissance de 1'état hydrique du sol et de son évolution temporelle et spatiale est une information importante dans la compréhension du fonctionnement hydrique (Ben Salem et al., 2013) et la phénologie (Rosnay et Baup, 2004) chez la plante. Suivant les saisons climatiques, la variation de la teneur en eau du sol dans la zone racinaire a été étudiée. Dans les deux sites, l'allure de la variation saisonnière montre une progression des teneur en eau du sol, de la SCC, où la disponibilité en eau du sol est très faible, en passant par la PSP, vers la SSF avec une plus grande disponibilité en eau. Egalement, plus le sol est profond, plus l'hmidité du sol est élevée. En effet, la connaissance de la disponibilité en eau du sol est impotante car la réponse des plantes par rapport à la sècheresse est complexe. Ainsi, les plantes développent une série de mécanismes morphologique, physiologique, biochimique, cellulaire et moléculaire, pour surmonter le déficit hydrique ou les conditions de stress de la sècheresse (Fang et Xiong, 2014). Insaloo et al., (2008) ont donné un résumé de quatre mécanismes de résistance à la sécheresse par les plantes que sont (i) l'échapement à la sècheresse, (ii) l'évitement à la sècheresse, (iii) la tolérence à la sècheresse, et (iv) la résistance aux sèvères stress hydiques à travers le mécanisme de survie.

D'après plusieurs auteurs (Fenner, 1980; Lobo et al., 2003; Van den Bilcke et al., 2013), le fait que le baobab est défeuillé durant une partie de la saison sèche, en laissant tomber ses feuilles très tôt, au début de la saison sèche, suggère l'exemple de la stratégie d'évitement à la sèheresse. Ces 
espèces qui répondent à cette stratégie effectuent un ajustement phénologique liées à une saison végétative précoce. Elles décalent leur saison de végétation, ou leur floraison et fructification afin qu'elles ne soient pas synchrones avec la période sèche (Zapater, 2009). Néamoins, ces espèces ne peuvent pas totalement échapper aux périodes de sècheresse (Brendel et Cochard, 2011). D’après ces derniers auteurs, les mécanismes développés par les arbres qui adoptent cette stratégie contribuent à limiter les pertes d'eau (fermeture des stomates et chute des feuilles) et à augmenter les entrée d'eau (enracinement profond), tout en réduisant leur croissance végétative durant la saison sèche.

Cependant, les observations phénologiques trouvées chez les baobabs de la zone d'étude en relation avec la variation saisonnière de l'humidité du sol corroborent bien la stratégie d'évitement à la sécheresse. En effet, c'est à la fin de la SSC que les baobabs du milieu présentent leurs premières feuilles au moment où l'humidité du sol est à son niveau le plus faible, bien avant les premières pluies. Cette précocité végétative observée sur le baobab a été déjà indiquée par Fenner (1980) et Chapotin et al. (2006a). Elle lui permet de décaler les phases de pleine feuillaison, de floraison et de fructification pour qu'elles ne coïncident pas avec la saison sèche. La période qui couvre la phase de feuillaison intense, de fin de la floraison et de début de la fructification correspond la PSP où le sol est suffisamment humide. Par contre, la période de début maturation des fruits et de la chute intense des feuilles est observée à partir de la fin de la saison des pluies qui précéde le début de la saison sèche. Egalement, cette adaptation par rapport aux saisons climatiques a été observée par Korbo et al. (2013) dans les jardins irrigués de jeunes baobabs dans le site de Sotuba en Bamako au Mali. D’après les résultats, la productivité moyenne en feuilles trouvée est plus élevée en saison des pluies ( de mai à septembre) avec $0,6 \mathrm{~kg} \cdot \mathrm{m}^{-2} \cdot \mathrm{mois}^{-1}$ comparée à celle en saison sèche (de novembre en mars) avec $0,2 \mathrm{~kg} \cdot \mathrm{m}^{-2} \cdot$ mois $^{-1}$. La productivité maximale est obtenue en juillet $\left(1,1 \mathrm{~kg} \cdot \mathrm{m}^{-2} \cdot \mathrm{mois}^{-1}\right)$ et celle minimale en janvier $\left(0,08 \mathrm{~kg} \cdot \mathrm{m}^{-2} \cdot \mathrm{mois}^{-1}\right)$.

\section{Variation saisonnière de l'état hydrique interne}

La variation de l'état hydrique interne saisonnière donne des potentiels hydiques foliaires et des teneurs relatives en eau plus élevés en début et en fin de la saison sèche contrairement à la saison pluvieuse. Ces observations sont en phase avec les espèces caduques à tronc succulent. Ces denières ont des potentiels hydriques des feuilles et des tiges très élevées en saison sèche, contrairement aux autres espèces caduques qui donnent des potentiels très bas (Brochert et $a l .$, 2002). En effet, le baobab est une espèce à tronc succulent (Collière, 2002; Chapotin et al., 2006a ; De Smedt et al., 2012; Van den Bilcke et al., 2013). Ce genre d'espéce est caduque et que pendant la saison sèche, leurs demandes en eau sont faibles (Chapotin et al., 2006b), de même que leurs 
pertes d'eau, avec également une grande capacité d'absorption et de conservation d'eau durant la saison des pluies.

En effet, le baobab adulte peut conserver d'importante quantité d'eau dans son tronc, plus de 120000 litres d'eau (Samba et al., 2003). Son bois fibreux a une grande proportion de cellules parenchymateuses qui constituent des réserves en eau (Wicken and Low, 2008). D'où une source d'importance d'eau pour les hommes et animaux pendant les conditions de sècheresse (Fenner,1980). Egalement, ces réserves d'eau jouent un rôle primordial dans le développement phénologique de l'espèce. Leurs fluctuations expriment en grande partie la variation hydrique interne du baobab. En effet, chez les espèces caduques à tronc succulent, les bourgeons végétatifs sont dormants durant la saison sèche (Brochert et al., 2002). Chez le baobab, leurs éclatements, qui s'accompagnent de l'expansion des premières feuilles, débutent en fin de la saison sèche avant même l'arrivée des premières pluies. Chapotin et al. (2006a) ont montré que cette expansion foliaire du baobab est due aux stocks d'eau du tronc de l'arbre. Mais, ces stocks d'eau ne permettent pas l'ouverture des stomates foliaires pour effectuer les échanges gazeux. La fermeture des stomates foliaires suggère un empêchement des pertes d'eau par transpiration foliaire par le maintien en turgescence des cellules au niveau de la feuille. Ceci pourrait alors justifier les potentiels hydriques foliaires élevés durant la fin de la saison sèche.

L'utilisation des stocks d'eau du tronc par le baobab en fin de la saison sèche pour le renouvellement de sa feuillaison pourrait être confirmée par le fait que le tronc des espèces caduques à tronc succulent se rétrécit pendant l'élatement des bourgeons végétatifs et n'augmente qu'après la première pluie (Daubainemire, 1972) dû probablement par les entrées d'eau à travers les racines. En effet, d'après Chapotin et al., (2006a), l'ouverture des stomates foliaires qui induit la perte d'eau par transpiration coïncide au début du flux de sève de la base du tronc après une pluie significative. La perte d'eau au niveau de la feuille entraîne la création de gradients de potentiel hydrique entre la feuille, la tige, l'appareil racinaire et le sol, qui expliquent largement l'ascension de l'eau dans la plante à partir du sol (Laffray et Louguet, 1991). En effet, l'absorption racinaire est contrôlée largement par la transpiration foliaire (Kozlowski, 1987).

Lorsque la quantité d'eau perdue par transpiration est supérieure à celle absorbée, les potentiels hydriques foliaires ainsi que les teneurs relatives en eau diminuent. En outre, Chapotin et al. (2006b) rapportent que lorsqu'il y a une forte demande en eau, les plantes à tronc succulent puisent sur leur stock d'eau pour ainsi éviter la forte pression dans le xylem pouvant conduire à une cavitation en permettant l'ouverture des stomates pour les échanges gazeux. De la même façon, pour le baobab, c'est en pleine saison de végétation, correspondant à la saison des pluies que les demandes en eau pour la 
croissance demeurent problablement très élevées. Egalement, l'anatomie de la plante de baobab témoigne, en plus de la présence de tissus de stockage d'eau, d'une vulnérabilité du xylem à l'embolie (Van den Bilcke et al., 2013) due par la faible densité de bois dans le tronc (Hacke et al., 2001).

La maturation, la sénescence et la chute des feuilles (avec des $\Psi$ élevés) des baobabs sont observées très tôt vers le début de la saison sèche au moment où l'humidité du sol est plus élevée dans la zone. En effet, Brochert et al. (2002) rapportent que chez les espèces à tronc succulent, les vieilles feuilles agées de six mois, avec régulièrement un potentiel hydique foliaire minimum élevé, deviennent sénescentes et tombent. Ceci indique que l'abcission de la feuille n'est pas causée par le stress hydrique comme l'attestent certains auteurs dont Fenner (1980) et Brochert et al. (2002). Ce derniers auteurs supposent que ce phénomène est dû par la diminution de la longueur du jour en conjonction de l'âge de la feuille. Par ailleurs la réponse chimique de l'acide abscissique (ABA) pourrait être à l'origine de la conservation de la turgescence des feuilles car il joue un rôle central dans les réponses physiologiques comme la fermeture stomatique et l'induction de la maturation (Lund et al., 2008).

\section{Effets des saisons climatiques sur l'état hydrique du baobab}

Les différentes saisons climatiques semblent être déterminantes sur les phases phénologiques du baobab ainsi que sur leurs variations hydriques internes. Car, il a été noté par Kabongo wa (2010) que les différentes saisons climatiques sont à l'origine des alternances des phases de repos et de reprise végétatives ainsi que le bon fonctionnement de leurs diverses manifestations phénologiques. En effet, les espèces végétales croissent en s'adaptant au climat de la zone dans lequel elles vivent. Ainsi, en période sèche, d'après Fenner (1980), la chute des feuilles des baobabs n'est pas directement causée par un stress hydrique. Selon lui, cette chute est mise en place pour réduire la croissance par la limitation de la photosynthèse au niveau des feuilles en cette période. Par ailleurs, d'après les travaux de Bouda et al., (2015), pour les jeunes plantes de baobab issues de semis, leurs paramètres de croissance reste faiblent durant les périodes des saisons fraiche et sèche, périodes durant lesquelles les baobabs adultes restent normalement sans feuilles. Egalement, durant cette période, avec Korbo et al., (2013), dans les jardins irrigués au Mali, les jeunes plantes de baobab donnent une faible production en feuilles. Cette adaptation végétative du baobab par rapport aux saisons climatiques a été également notée chez les espèces caduques à tronc succulent, dans la forêt sémi-caduque Guanacaste au Costa Rica où les pluies anormales $(70 \mathrm{~mm})$ qui se sont produites en mi-saison sèche (1998/1999) avaient provoqué l'éclatement des bourgeons et l'épanouissement des feuilles de l'ensemble des arbres caduque à l'exception de ceux à tronc succulent (Brochert et al., 2002). 
Malgré l'état hydrique interne du baobab qui évolue saisonnièrement de façon différente par rapport à d'autres espèces végétales, l'effet induit par les saisons climatiques trouvé statistiquement sur les variables hydriques corrobore certains travaux. En effet, D’Andria et al. (2009) ont trouvé une diminution progressive des potentiels hydriques ( $\Psi \mathrm{b}$ et $\Psi \mathrm{m})$ et de façon concomitante avec des sécheresses saisonnières sur Olea europaea L.(olivier). Egalement, Eyog-Matig et Dreyer (1991) ont montré chez quatre espèces végétales forestières utilisées dans les reboisements au Nord - Cameroun (Dalbergia sissoo, Khaya seneglensis, Azadirachta indica et Eucalyptus camaldulensis), que leur $\Psi$ présente de fortes variations. Il oscille de -0,3 Mpa (-3bars) en saison humide à -3,5 Mpa (-35bars) en saison sèche.

\section{Effet de la toposéquence}

L'effet induit par les saisons climatiques sur l'état hydrique interne du baobab est bien réel et prouvé statistiquement par les résultats à l'échelle de l'ensemble des baobabs de la zone d'étude en général et en particulier de ceux situés sur le plateau. Par contre, à l'échelle des baobabs situés dans le basfond, cet effet est masqué. Ceci pourrait être lié aux conditions hydriques du sol plus humides sur le bas-fond avec la présence de la vallée contrairement au plateau. Pour corroborer cette réalité, Yao et Amadou (1988) ont montré l'importance du lac de Kossou (Côte d'Ivoire) sur l'humidité du sol en condition hydrique défavorable. Au point de flétrissement, les valeurs de humidité du sol proche du lac sont supérieures à celles qui lui sont éloignées. Egalement, Zine El Abidine et al., (2016) ont posé l'hypothèse selon laquelle, la présence de nappe phréatique, proche au niveau de la vallée de Oued N'fis, permettrait de compenser les pertes d'eau par transpiration due au climat aride chez le cyprès de l'Atlas.

\section{Conclusion}

Les résultats ont permis de mieux appréhender le fonctionnement hydrique de $A$. digitata L. qui semble être conditionné par d'autres facteurs que la disponibilté de l'eau du sol. En effet, contrairement à la variation de l'humidité du sol, le potentiel hydrique foliaire et la teneur relative en eau indiquent un état hydrique interne du baobab plus élevé en saison sèche (fin saison des pluies et fin saison sèche) contrairement en pleine saison pluvieuse. D'où le rôle primordial que jouent les saisons climatiques. Egalement, la toposéquence agit sur l'état hydrique du baobab en minimisant sa variation saisonnière sur le bas-fond contrairement à ce qui est observé sur la plateau. En outre, les observations phénologiques du baobab montrent un cycle phénologique de stratégie d'évitement à la sècheresse.

Par ailleurs, ces résultats justifient la capacité d'adaptation phénologique du baobab avec les saisons climatiques et sa tolérence à la 
sécheresse saisonnière du sol. Egalement, ils montrent son aptitude de pouvoir subsister sur un bas-fond ou un plateau. Ces résultats devront permettre de mieux encourager la propagation de plantation du baobabs dans le contexte de dégradation de la ressource et des changements climatiques.

\section{Remerciement}

Nous adressons nos sincères remerciements au FNRAA/WAAPP qui nous a attribué une allocation de recherches à travers l'ISRA.

Nous exprimons également toute notre gratitude à l'ensemble des personnes du CNRF, des autres centres de l'ISRA et de l'Université Assane Seck de Ziguinchor (UASZ) qui ont apporté leurs soutients.

\section{References:}

1. Achetar M., Rambal S., Lepart J., 1991. Evolution du potentiel hydrique foliaire et de la conductance somatique de quatre chênes méditerranéens lors d'une période de dessèchement. Annales des sciences forestières, INRA/EDP Sciences, 1991, 48(5), pp.561-573. $<$ hal-00882774>.

2. Bationo B.A., Ouédrago S.J., Alexandre D., Guinko S., 2001. Statut hydrique de quatre espèces ligneuses. Soudaniennes dans la forêt de Nazinon, Burkina Faso in Sécheresse n² vol. 12, juin 2001. 87-94pp.

3. Bationo B.A., Lamien N., Dermes N., Kandji S., 2009. Culture du baobab, Adansonia digitata L. (bombacaceae) en planche maraîchère : une méthode pour simplifier sa récolte et favoriser sa propagation au sahel in Bois et Forêts des Tropiques, 2009, N99(1) : 79-86pp.

4. Ben Salem A., Majdoub R., M'Sadaky et Boujnah D., 2013. Impact de l'état Hydrique du Sol sur le Comportement Éco-physiologique d'une Oliveraie Adulte Aménagée en Meskat (Sahel Tunisien). Algerian journal of arid environment 14 vol. 3, n 2, Décembre 2013: 4-14pp.

5. Bouda ZH-N, Bayala J, Markussen B, Jensen JS, Ræbild A 2015. Reactions of Adansonia digitata L. provenances to long-term stress at seedling stage. Agroforest Syst (2015) 89:113-123. DOI 10.1007/s10457-014-9746-x.

6. Bourou S., 2012. Etude éco-physiologique du tamarinier (Tamarindus indica L.) en milieu tropical aride. Thèse de doctorat $(\mathrm{PhD})$, faculté des sciences en Bio-ingénierie, Université de Grand, Belgique, 164p.

7. Brendel O, Cochard H. 2011. How plant species cope with water stress. In: Birot Y, Gracia C, Palahi M. eds. Water for forest and people in the Mediterranean: a challenging balance. European Forest Institute, 76-80. 
8. Brochert R., Rivera G. and Hagnauer W. 2002. Modification of Vegetative Phenology in a Tropical Semi-deciduous Forest by Abnormal Drought and Rain. Biotropica 34(1): 27-39 2002.

9. Cissé M., 2015. Analyse des perceptions locales et des déterminants socio-économiques de l'adoption de technologies agroforestières : Cas des planches maraichères et du greffage horticole d'Adansonia digitata L. (Baobab) en Moyenne et Haute Casamance, Sénégal. Mémoire de licence, Université Assane SECK de Ziguinchor, 57p.

10. Chapotin, S.M., Razanameharizaka, J.H., Holbrook, N.M., $2006 a$. Baobab trees (Adansonia) in Madagascar use stored water to flush new leaves but not to support stomatal opening before the rainy season. New Phytologist 169, 549-559.

11. Chapotin, S.M., Razanameharizaka, J.H., Holbrook, N.M., 2006b. Water relations of baobab trees (Adansonia spp. L.) during the rainy season: does stem water buffer daily water deficits? Plant Cell and Environment 29, 1021-1032.

12. Chapotin, S.M., Razanameharizaka, J.H., Holbrook, N.M., 2006c. A biomechanical perspective on the role of large stem volume and high water content in baobab trees (Adansonia spp.; Bombacaceae). American Journal of Botany 93, 1251-1264.

13. Collière P., 2002. La forêt de Baobab (Adansonia digitata L.) de Nguekhokh (Sénégal): analyse des causes de la dégradation de la forêt et recherche de solutions In chaîne et Baobab, Rapport d'étude de la mission humanitaire réalisée au CIAF de Nguekhokh du 27 juin au 21 août 2002 par Pierre Collière, 34pp

14. D’Andria R., Lavini A., Morelli G., Sebastiani L. \& Tognetti R. 2009. Physiological and productive responses of Olea europaea L. cultivars Frantoio and Leccino to a regulated deficit irrigation regime. Plant Biosystems, 143 (1), 222-231.

15. Daubenmire R. 1972. Phenology and other characteristics of tropical semi-deciduous forest in north-western Costa Rica. Journal of Ecology 60: 147-170.

16. De Smedt, S., Cuní Sanchez, A., Van den Bilcke, N., Simbo, D., Potters, G., Samson, R., 2012. Functional responses of baobab (Adansonia digitata L.) seedlings to drought conditions: Differences between western and south-eastern Africa. Environmental and Experimental Botany 75, 181-187.

17. Diagne et A.L. 2000. Influence d'un déficit pluviométrique sur le fonctionnement hydique foliaire de Acacia tortilis en zone semi-aride (Ferlo, Nord Sénégal), Thèse de doctorat de $3^{\mathrm{e}}$ cycle ISE, UCAD, Dakar, 111p. 
18. Eyog-Matig O., Dreyer E., 1991. Fonctionnement hydrique de quatre essences de reboisement au Nord-Cameroun : évolution saisonnière du potentiel hydrique et de la conductance somatique. In Physiologie des Arbres et Arbustes en zone arides et semi-arides : 85-91.

19. Fahmi F., Tahrouch S., Bouzoubaa Z., Hatimi A., 2011. Effet de l'aridité sur la biochimie et la physiologie de d'Argania sponosa. Acte du Premier Congrès International de l'Arganier, Agadir 15-17 décembre 2011, 299-308pp.

20. Fang Y. and Xiong L. 2015. General mechanisms of drought response and their application in drought resistance improvement in plants. Cellular and Molecular Life Sciences CMLS (2015) 72:673-689. DOI 10.1007/s00018-014-1767-0.

21. Fenner M., 1980. Some Measurements on the Water Relations of Baobab Trees. Biotropica 12(3): 205-209.

22. Hacke U.G., Sperry J.S., Pockman W.T., Davis S.D. \& McCulloh K.A. (2001) Trends in wood density and structure are linked to prevention of xylem implosion by negative pressure. Oecologia 126, 457-461.

23. Izanloo A., Condon A. G., Langridge P., Tester M. and Schnurbusch T. 2008. Different mechanisms of adaptation to cyclic water stress in two South Australian bread wheat cultivars. Journal of Experimental Botany, Vol. 59, No. 12, pp. 3327-3346.

24. Jabot F., 2009. Marches aléatoires en forêt tropicale. Contribution à la théorie de la biodiversité. Thèse de doctorat de l'université de Toulouse III-Paul Sabatier, Spécialité : Ecologie, Biodiversité et Evolution, 269p.

25. Kabongo wa T. T., 2010. Etude corrélative entre la phénologie du Pericopsis elata HARMS et les paramètres écoclimatiques dans la région de Yangambi en République Démocratique du Congo. GeoEco-Trop., 2010, 34: 127 - 138.

26. Korbo, A., Kjær, E. D., Sanou, H., Ræbild, A., Jensen, J. S., \& Hansen, J. K. (2013). Breeding for high production of leaves of baobab (Adansonia digitata L.) in an irrigated hedge system. Tree Genetics and Genomes, 9, 779-793. doi: 10.1007/s11295-013-0595-y.

27. Kozlowski, T.T., 1987. Soil moisture and absorption of water by tree roots. J. Arboric. 13(2): 39-46.

28. Laffray, D. et Louguet, P. (1991). Physiologie des arbres et des arbustes en zones arides et semi-aride: L'appareil stomatique et la résistance à la sécheresse chez les végétaux ligneux. Groupe d'étude de l'arbre, Paris, pp. 21-46.

29. Lobo JA, Quesada M, Stoner KE, Fuchs EJ, Herrerías-Diego Y, Rojas J, Saborío G. 2003. Factors affecting phenological patterns of 
Bombacaceous trees in seasonal forests in Costa Rica and Mexico. American Journal of Botany 90: 1054-1063.

30. Lund S.T., Peng F.Y., Nayar T., Reid K.E., Schlosser J., 2008. Gene expression analyses in individual grape Vitis vinifera L.) berries during ripening initiation reveal that pigmentation intensity is a valid indicator of developmental staging within the cluster. Plant Molecular Biology 68, 301-315.

31. Mukhtar, R.B. 2016. Effect of drought stress on early growth of adansonia digitata (L.) In semi-arid region of Nigeria. Journal of Research in Forestry, Wildlife \& Environment Vol. 8(4) December, 2016 http://www.ajol.info/index.php/jrfwe.

32. Ndiaye A., 2013. Caractérisation biophysique des parcs à Adansonia digitata L. (Baobab) en Moyenne et Haute Casamance : dynamique, production fruitière et anthropisation. Mémoire master 2, Département Agroforesterie Université Assane Seck de Ziguinchor, 68p.

33. Randriamanana, T.,Wang, F., Lehto, T., Aphalo, P.J., 2012.Water use strategies of seedlings of three Malagasy Adansonia species under drought. South African Journal of Botany 81, 61-70.

34. Rosay P. et Baup F., 2004. Mesure d'humidité du sol au Gourma, 14p.

35. Sagna P., 2005. Dynamique du climat et son évolution récente dans la partie ouest de l'Afrique occidentale. Thèse de doctorat, faculté des lettres et sciences humaines, Département de Géographie, Université Cheikh Anta Diop Dakar. 790p.

36. Samba N.A.S., Gaye, A., Fall S.T., Diallo I., (2003). Le baobab, Nouvelle plante maraîchère du Sahel, fiche technique.

37. Savard V., (2003). Evaluation du potentiel d'adoption des parcelles maraîchères de baobab (Adansonia digitata) dans la région de Ségou, au Mali, mémoire de Master Université de Laval, Octobre 2003. 127p.

38. Van den Bilcke, N., De Smedt, S., Simbo, D.J., Samson, R., 2013. Sap flow and water use in African baobab (Adansonia digitata L.) seedlings in response to drought stress. South African Journal of Botany. http://dx.doi.org/10.1016/j.sajb.2013.09.006.

39. Wickens GE, Lowe P (2008) The baobabs: Pachycauls of Africa, Madagascar and Australia. Springer, New York. ISBN 978-1-40206430-2. OCLC 166358049.

40. Yao N.R., Amadou O., 1988. Humidité du sol le long de la toposéquence du Lac de Kossou : profils et variation de stocks hydriques d'un sol ferrallitique. In : Milieux poreux et transferts hydriques. Bulletin du Groupe Français d'Humidimétrie Neutronique, (23), 39-52. ISSN 0245-9493.

41. Zapater M., 2009. Diversité fonctionnelle de la réponse à la sécheresse édaphique d'espèces feuillues en peuplement mélangé : Approches 
écophysiologique et isotopique. Thèse de doctorat en Biologie forestière. Sous la direction d'André Granier. Soutenue le 10-11-2009 à Nancy $1.310 \mathrm{p}$.

42. Zine El Abidine A., Bouderrah M., Moustahssen A., Lamhamedi M. S. 2016, .Relations hydriques et croissance de plants soumis à un déficit hydrique édaphique graduel. Cas du cèdre de l'Atlas, du pin maritime de montagne et du cyprès de l'Atlas. Forêt Mediterranéenne, t. XXXVII, $n^{\circ}$ 4, décembre 2016, pp : 327-342 